University of Nebraska - Lincoln

DigitalCommons@University of Nebraska - Lincoln

Gordon Gallup Publications

Research Papers in Physics and Astronomy

September 1966

On the Symmetry of Slater Determinantal Wavefunctions

Gordon A. Gallup

UNL,ggallup1@unl.edu

Follow this and additional works at: https://digitalcommons.unl.edu/physicsgallup

Part of the Physics Commons

Gallup, Gordon A., "On the Symmetry of Slater Determinantal Wavefunctions" (1966). Gordon Gallup Publications. 24.

https://digitalcommons.unl.edu/physicsgallup/24

This Article is brought to you for free and open access by the Research Papers in Physics and Astronomy at DigitalCommons@University of Nebraska - Lincoln. It has been accepted for inclusion in Gordon Gallup Publications by an authorized administrator of DigitalCommons@University of Nebraska - Lincoln. 


\title{
On the Symmetry of Slater Determinantal Wavefunctions
}

\author{
G. A. GalluP \\ Department of Chemistry, University of Nebraska, Lincoln, Nebraska
}

(Received 20 May 1966)

\begin{abstract}
A method involving the use of characteristic polynomials of irreducible representation matrices of groups and yielding the symmetry species of Slater determinantal functions is given. The necessary polynomials have been calculated for the commonly occurring symmetry groups and are listed to supplement the usual character tables.
\end{abstract}

\section{INTRODUCTION}

$\mathbf{T}$ IHE use of the theory of group representations to simplify quantum-mechanical calculations is so well known as to require no review. ${ }^{1}$ Nevertheless, a brief resumé of the steps in a frequently used procedure is useful as a preface to this discussion.

After the group of covering operations for the Hamiltonian has been determined, linear combinations belonging to definite symmetry species are formed from some set of basis functions. Frequently, these are oneelectron functions to be used for constructing Slater determinantal functions (SDF) to represent approximate, antisymmetric states of the complete $n$-electron system. The symmetry species of the determinantal functions can be elucidated in a straightforward manner by calculating the characters and performing the usual species analysis. The determinations of these characters can be quite arduous, however, particularly for excited states of systems having degenerate symmetry species.

This article discusses a fairly simple algorithm which can be used to determine the symmetry species of determinantal functions from the species of the occupied one-electron orbitals. This procedure does not determine the actual linear combinations belonging to each species, but knowing in advance which species projection operators to use can save considerable labor.

\section{DERIVATION OF ALGORITHM}

\section{A. Spin-Free Case}

The system is assumed to have symmetry species $\Gamma_{1}, \Gamma_{2}, \Gamma_{3}, \cdots, \Gamma_{k}$, not necessarily distinct, which are partially or completely filled with electrons in the Slater determinantal function (SDF). The configuration is then $\Gamma_{1}^{n_{1}} \Gamma_{2}^{n_{2}} \cdots \Gamma_{k}^{n_{k}}$ with $n_{i}$ the number of electrons occupying the $i$ th symmetry species. For an SDF of $n$ electrons, we have $n=\sum_{i} n_{i}$. If the degeneracy of $\Gamma_{i}$ is $g_{i}$ and the possibility of both spin orientations is included, the number of linearly independent SDF in the configuration is $G=\prod_{i}\left[\left(2 g_{i}\right) ! / n_{i} !\left(2 g_{i}-n_{i}\right) !\right]$, which is, of course, the familiar Fermi-Dirac counting formula. If interest is centered on SDF, specified so

${ }^{1}$ (a) E. P. Wigner, Group Theory (Academic Press Inc., New York, 1959); (b) H. Eyring, J. Walter, and G. E. Kimbal, Quantum Chemistry (John Wiley \& Sons, Inc., New York, 1944). that among the electrons $n_{i}$ in $\Gamma_{i}, p_{i}$ have $m_{S}$ values of $+\frac{1}{2}$ and $n_{i}-p_{i}$ have $m_{S}$ values of $-\frac{1}{2}$, the total $M_{S}$ value is then $\left(\frac{1}{2}\right) \sum_{i}\left(2 p_{i}-n_{i}\right)$. The number of linearly independent SDF in the configuration of this type is

$$
G(p)=\prod_{i}\left[\frac{g_{i} !}{p_{i} !\left(g_{i}-p_{i}\right) !} \frac{g_{i} !}{\left(n_{i}-p_{i}\right) !\left(g_{i}+p_{i}-n_{i}\right) !}\right] .
$$

If one of the $G(p) \mathrm{SDF}$ is subjected to transformation by $\mathcal{R}$, one of the covering operations of the group, a linear combination of the SDF is obtained. If the $R$ are assumed to be spin free, $M_{S}$ is unchanged in the process. If $\Psi(p)$ denotes the subset designated by $p_{i}$ of all the SDF belonging to the configuration, $\Psi(p) \rightarrow$ $\mathcal{\Psi}(p)$ is a linear transformation represented by a $G(p) \times G(p)$ matrix. The trace (character) of the matrix is needed.

In order to calculate the trace, the effect of $R$ on one of the symmetry species is determined. The $p_{i}$ spin orbitals from $\Gamma_{i}$ with $m_{S}=\frac{1}{2}$ are transformed among themselves by $\mathcal{R}$, since these functions are assumed to form a basis for an irreducible representation of the group. The spin orbitals are denoted $u_{i}(j) \alpha, j=1,2$, $\cdots, g_{i} . p_{i}$ of these are involved in any one SDF. Thus, one obtains

$$
\Re u_{i}(j) \alpha=\sum_{k} D_{i}(R)_{k j} u_{i}(k) \alpha .
$$

If the SDF contains $u_{i}\left(j_{1}\right) \alpha, u_{i}\left(j_{2}\right) \alpha, \cdots, u_{i}\left(j_{p_{i}}\right) \alpha$ and similarly $u_{i}\left(k_{i}\right) \beta, u_{i}\left(k_{2}\right) \beta, \cdots, u_{i}\left(k_{n_{i}-p_{i}}\right) \beta$, the diagonal element of the matrix giving the linear transformation $R \Psi(p)$ is a product of determinants obtained from the irreducible-representation matrices $D_{i}(\Omega)$. From $\Gamma_{i}$ we obtain the product of two determinants: one is the $p_{i} \times p_{i}$ determinant constructed from the $j_{1}$ row and column, $j_{2}$ row and column, $\cdots, j_{p_{i}}$ row and column of $D_{i}(R)$. The other determinant is the similarly constructed one from the $m_{S}=-\frac{1}{2}$ functions with $n_{i}-p_{i}$ rows and columns. If we sum over the $\left[g_{i} ! / p_{i} !\left(g_{i}-p_{i}\right) !\right]$ diagonal elements of the linear transformation $\mathfrak{R} \Psi(p)$ corresponding to all the possible ways that the $u_{i}(j) \alpha$ may be occupied, it is easily seen that the contribution from $\Gamma_{i}, m_{S}=+\frac{1}{2}$ states is just $(-1)^{g_{i}-p_{i}}$ times the coefficient of $\mu^{g_{i}-p_{i}}$ in the characteristic polynomial for $D_{i}(\mathcal{R})$ :

$$
Q_{i}(R, \mu)=\left|D_{i}(\mathcal{R})-\mu I\right| \text {. }
$$


It is more convenient below to have the polynomials arranged so that the exponent corresponds to the number of electrons rather than the $g_{i}$ complement and to have the signs all positive, therefore a new indeterminant $\lambda=1 / \mu$ is introduced and $Q_{i}$ is transformed:

$$
P_{i}(\Re, \lambda)=\lambda^{\sigma} Q_{i}(\Re,-1 / \lambda)=\left|\lambda D_{i}(\Re)+I\right| \text {. }
$$

Since the characteristic polynomial depends only upon the eigenvalues of a matrix, and each group-representation element belonging to a given class has the same eigenvalues, $P_{i}(R, \lambda)$ will be a class function of the group. The coefficients of a given power of $\lambda$ in all of the $P_{i}(R, \lambda)$ form a character of a reducible or irreducible representation of the group, so the results could be summarized by giving one polynomial with the coefficient of $\lambda^{i}$ written as the appropriate symmetry species (irreducible representation) or sum of symmetry species. The argument $R$ may now be suppressed from the symbol $P_{i}(R, \lambda)$.

Once the $P_{i}(\lambda)$ have been determined for each occupied symmetry species of the SDF, the composite symmetry is easily determined by forming the product

$$
P=\prod_{i=1}^{k} P_{i}\left(\lambda_{i}\right) P_{i}\left(\lambda_{i}{ }^{\prime}\right),
$$

where species multiplication as determined by the group is used to evaluate the new coefficients. The coefficient of $\lambda_{1}{ }^{p_{1}}\left(\lambda_{1}{ }^{\prime}\right)^{n_{1-p} p_{1}} \lambda_{2}{ }^{p_{2}}\left(\lambda_{2}{ }^{\prime}\right)^{n_{2}-p_{2}} \ldots$ in $P$ gives the symmetry species contained in the original SDF.

In general discussions of the theory of group representations ${ }^{1 \mathrm{~b}}$ it is customary to collect together a number of results in the form of character tables. Such collections do not usually contain the characteristic polynomials of the irreducible representation matrices. Therefore, the $P_{i}(\lambda)$ are given in Appendix $\mathrm{A}$ for the degenerate representations of the point groups commonly given in such listings. The one dimensional representations can be obtained immediately, since in this case the character and the eigenvalue are the same.

\section{B. Generalization to Include Spin}

The foregoing discussion would not give particularly useful results unless it could be generalized to include the computation of the correct spin states. This is easily done by calculating the characteristic polynomials associated with the product group formed from the point group appropriate for the space part of the spin orbitals and the two-dimensional unimodular, unitary group appropriate for the spin functions. This is easily done by examining the eigenvalues of a group element in each class. Again the results may be summarized by giving a single polynomial with the species notation given for each coefficient. The spin states are given by the usual anterior superscript $2 S+1$. If these new polynomials are denoted $R_{i}(\lambda)$, the correct symmetry and spin states are given by the coefficient of $\lambda_{1}{ }^{n} \lambda_{2}{ }^{{ }^{2}} \lambda_{3}{ }^{{ }^{3}} \ldots$ in the product

$$
R=\prod_{i=1}^{k} R_{i}\left(\lambda_{i}\right)
$$

where we have obtained $R_{i}(\lambda)$ from the characteristic polynomial by a transformation similar to $Q_{i} \rightarrow P_{i \text {. }}$. Again, the coefficients in $R$ are computed from the species product rules of the group of the space functions and the standard decomposition formula for angular momentum,

$$
\begin{aligned}
(2 S+1) & \times\left(2 S^{\prime}+1\right) \\
= & {\left[2\left(S+S^{\prime}\right)+1\right],\left[2\left(S+S^{\prime}\right)-1\right], \cdots, } \\
& {\left[2\left|S-S^{\prime}\right|+1\right] . }
\end{aligned}
$$

The polynomials $R_{i}(\lambda)$ are also given in Appendix A for the degenerate species of the common point groups. The foregoing discussion applies equally well to both continuous and discrete groups and Appendix $B$ gives a method of generating the $R_{i}(\lambda)$ for the atomic case of Russel-Saunders coupling.

\section{EXAMPLES}

Although the foregoing discussion is fairly selfexplanatory, examples might be useful to clarify the procedure. We consider the following cases.

\section{A. Some Excited States of Benzene, $D_{6 h}$}

If the Hückel molecular orbitals for the $\pi$ system of benzene have been determined in the standard fashion, the symmetry species of the one-electron functions are $A_{2 u}, E_{1 g}, E_{2 u}, B_{2 g}{ }^{2}$ The configurations $A_{2 u}{ }^{2} E_{1 g}{ }^{4-i} E_{2 u}{ }^{i}$, $i=0,1,2,3,4$ may be obtained by examining the product of the $R$ polynomials corresponding to the $A_{2 u}, E_{18}$, and $E_{2 u}$ species. We get from Table IV in Appendix A

$$
\begin{gathered}
{\left[{ }^{1} A_{1_{g}}\left(\lambda_{1}{ }^{2}+1\right)+{ }^{2} A_{2 u} \lambda_{1}\right]\left[{ }^{1} A_{1 g}\left(\lambda_{2}{ }^{4}+1\right)+{ }^{2} E_{1 g}\left(\lambda_{2}{ }^{3}+\lambda_{2}\right)\right.} \\
\left.+\left({ }^{3} A_{2 g}+{ }^{1} A_{1 g}+{ }^{1} E_{2 g}\right) \lambda_{2}{ }^{2}\right]\left[{ }^{1} A_{1 g}\left(\lambda_{3}{ }^{4}+1\right)+{ }^{2} E_{2 u}\left(\lambda_{3}{ }^{3}+\lambda_{3}\right)\right. \\
\left.+\left({ }^{3} A_{2 g}+{ }^{1} A_{1 g}+{ }^{1} E_{2 g}\right) \lambda_{3}{ }^{2}\right]={ }^{1} A_{1 g}\left(\lambda_{1}{ }^{2} \lambda_{2}{ }^{4}+\lambda_{1}{ }^{2} \lambda_{3}{ }^{4}\right) \\
+\left({ }^{3} B_{1 u}+{ }^{3} B_{2 u}+{ }^{3} E_{1 u}+{ }^{1} B_{1 u}+{ }^{1} B_{2 u}+{ }^{1} E_{1 u}\right) \\
\times\left(\lambda_{1}{ }^{2} \lambda_{2}{ }^{3} \lambda_{3}+\lambda_{1}{ }^{2} \lambda_{2} \lambda_{3}{ }^{3}\right)+\left({ }^{5} A_{1 g}+{ }^{3} A_{1 g}+2^{3} A_{2 g}\right. \\
\left.+2^{3} E_{2 g}+3{ }^{1} A_{1 g}+{ }^{1} A_{2 g}+3^{1} E_{2 g}\right) \lambda_{1}{ }^{2} \lambda_{2}{ }^{2} \lambda_{3}{ }^{2} \\
+(\text { terms with sum of exponents } \neq 0) .
\end{gathered}
$$

The coefficient of $\lambda_{1}{ }^{2} \lambda_{2}{ }^{4-i} \lambda_{3}{ }^{i}$ gives the states present in the configurations. The terms in the polynomial not given correspond to configurations of ionized benzene.

\section{B. Atomic Configuration $d^{2}$}

From Table VII in Appendix B we find that the characteristic polynomial for equivalent $d$ (which are

${ }^{2}$ G. W. King, Spectroscopy and Molecular Structure (Holt, Rinehart and Winston, Inc., New York, 1964). 
TABLe I. Polynomials $P$ for two-dimensional representations of common, finite point groups and $C_{\infty v}$ and $D_{\infty h}$.

\begin{tabular}{|c|c|c|c|c|}
\hline Group & Species & $\lambda^{2}$ & $\lambda$ & $\lambda^{0}$ \\
\hline$D_{3}, C_{z v}$ & $E$ & $A_{2}$ & $E$ & $A_{1}$ \\
\hline$D_{4}, C_{40}$ & $E$ & $A_{2}$ & $E$ & $A_{\mathrm{I}}$ \\
\hline$D_{5}, C_{b v}$ & $E_{\boldsymbol{i}}$ & $A_{2}$ & $E_{i}$ & $A_{1}$ \\
\hline$D_{6}, C_{6 v}$ & $E_{\boldsymbol{i}}$ & $A_{2}$ & $E_{i}$ & $A_{1}$ \\
\hline$D_{3 h}$ & $E^{(p)}$ & $A_{2}^{\prime}$ & $E^{(p)}$ & $A_{1}^{\prime}$ \\
\hline$D_{4 h}$ & $E_{g(u)}$ & $A_{2 g}$ & $E_{g(u)}$ & $A_{10}$ \\
\hline$D_{5 h}$ & $E_{i}^{(p)}$ & $A_{2}^{\prime}$ & $E_{i}^{(p)}$ & $A_{1}^{\prime}$ \\
\hline$D_{6 h}$ & $E_{i g(u)}$ & $A_{2 g}$ & $E_{i g(u)}$ & $A_{1 g}$ \\
\hline$D_{2 d}$ & $E$ & $A_{2}$ & $E$ & $A_{1}$ \\
\hline$D_{3 d}$ & $E_{g(u)}$ & $A_{2 \mathrm{v}}$ & $E_{g(u)}$ & $A_{1 g}$ \\
\hline$D_{4 d}$ & $E_{i}$ & $A_{2}$ & $E_{i}$ & $A_{1}$ \\
\hline$D_{5 d}$ & $E_{i g(u)}$ & $A_{2 \sigma}$ & $E_{i g(u)}$ & $A_{1 \sigma}$ \\
\hline$D_{6 d}$ & $E_{i}$ & $A_{2}$ & $E_{i}$ & $A_{1}$ \\
\hline$T_{d}, O$ & $E$ & $A_{2}$ & $E$ & $A_{1}$ \\
\hline$O_{h}$ & $E_{o(u)}$ & $A_{2 \sigma}$ & $E_{g(u)}$ & $A_{1 \sigma}$ \\
\hline$C_{v}^{\mathrm{a}}$ & $\Lambda$ & $\Sigma^{-}$ & $\Lambda$ & $\mathbf{s}^{+}$ \\
\hline$D_{\mathrm{coh}}^{\mathrm{A}}$ & $\Lambda_{g(u)}$ & $\Sigma_{\sigma}^{-}$ & $\Lambda_{g(u)}$ & $\Sigma_{0}^{+}$ \\
\hline
\end{tabular}

a stands for $\Pi, \Delta, \Phi$, etc.

even) electrons is given by

\section{$G_{0}{ }^{e} G_{1}{ }^{e} G_{2}{ }^{e}$}

$$
\begin{aligned}
=\left[{ }^{1} S^{e}\right. & \left.\left(\lambda^{2}+1\right)+{ }^{2} S^{e} \lambda\right]\left[{ }^{1} S^{e}\left(\lambda^{4}+1\right)+\left({ }^{2} P^{e}-{ }^{2} S^{e}\right)\left(\lambda^{3}+\lambda\right)\right. \\
& \left.+\left({ }^{3} S^{e}+{ }^{1} S^{e}+{ }^{1} D^{e}-{ }^{1} P^{e}\right) \lambda^{2}\right]\left[{ }^{1} S^{e}\left(\lambda^{4}+1\right)\right. \\
& \left.+\left({ }^{2} D^{e}-{ }^{2} P^{e}\right)\left(\lambda^{3}+\lambda\right)+\left({ }^{3} S^{e}+{ }^{1} S^{e}+{ }^{1} G^{e}-{ }^{1} F^{e}\right) \lambda^{2}\right] .
\end{aligned}
$$

The coefficient of $\lambda^{2}$ (and $\lambda^{8}$ ) in this polynomial is

$$
{ }^{1} S^{e}+{ }^{1} G^{e}+{ }^{3} F^{e}+{ }^{3} P^{e}+{ }^{1} D^{e} .
$$

This result is very familiar, of course, being obtainable by several other methods.

\section{APPENDIX A}

This appendix collects the results (Tables I-VI) for the Polynomials $P_{i}$ and $R_{i}$ for the degenerate species of the common groups. As was mentioned above, $P_{i}$ and $R_{i}$ may be written down by inspection for one-dimensional species. Thus, if $X$ is the species notation for any

\begin{tabular}{|c|c|c|c|c|c|}
\hline Group & Species & $\lambda^{3}$ & $\lambda^{2}$ & $\lambda$ & $\lambda^{0}$ \\
\hline$T$ & $F$ & $A$ & $F$ & $F$ & $A$ \\
\hline$T_{h}$ & $F_{\theta(u)}$ & $A_{\sigma(u)}$ & $F_{g}$ & $F_{o(u)}$ & $A_{0}$ \\
\hline$T_{d}, O$ & $F_{i}$ & $A_{i}$ & $F_{1}$ & $F_{i}$ & $A_{1}$ \\
\hline$O_{k}$ & $F_{i o(u)}$ & $A_{i g(u)}$ & $F_{1 q}$ & $F_{i v(u)}$ & $A_{1 v}$ \\
\hline$I$ & $F_{i}$ & $A$ & $F_{i}$ & $F_{i}$ & $A$ \\
\hline$I_{h}$ & $F_{i v(u)}$ & $A_{\theta(u)}$ & $F_{i v}$ & $F_{i g(u)}$ & $A_{\mathrm{g}}$ \\
\hline
\end{tabular}
one-dimensional species of any group and $A$ the symbol

Table II. Polynomials $P$ for three-dimensional representations of Point Groups $T, T_{d}, O_{h}, I$, and $I_{h}$. for the totally symmetric species,

and

$$
P=X \lambda+A
$$

$$
R={ }^{1} A\left(\lambda^{2}+1\right)+{ }^{2} X \lambda \text {. }
$$

\section{APPENDIX B}

Herein is given a method for obtaining $R$ polynomials for the product group of the three-dimensional rotation group $R_{\mathbf{3}}$ with the two-dimensional unimodular unitary group $U_{2}$ for application to Russell-Saunders states for atomic configurations. The fact that these groups are homomorphic does not enter into the present calculations. The method is given in some detail since a simple modification was used to obtain the results listed in Appendix A.

\begin{tabular}{|c|c|c|}
\hline \multirow[t]{2}{*}{$I:$} & $G:$ & $A \lambda^{4}+G \lambda^{3}+\left(F_{1}+F_{2}\right) \lambda^{2}+G \lambda+A$ \\
\hline & $H:$ & $A \lambda^{6}+H \lambda^{4}+\left(F_{1}+F_{2}+G\right)\left(\lambda^{3}+\lambda^{2}\right)+H \lambda+A$ \\
\hline \multirow[t]{2}{*}{$I_{h}:$} & $G_{\sigma(u)}:$ & $A_{g} \lambda^{4}+G_{g(u)} \lambda^{8}+\left(F_{1 \sigma}+F_{2 g}\right) \lambda^{2}+G_{o(u)} \lambda+A_{v}$ \\
\hline & $H_{\sigma(u)}:$ & $\begin{array}{l}A_{\sigma(u)} \lambda^{5}+H_{g} \lambda^{4}+\left(F_{1 g(u)}+F_{2 g(u)}+G_{o(u)}\right) \lambda^{3} \\
\quad+\left(F_{1 q}+F_{2 \sigma}+G_{g}\right) \lambda+H_{q(u)} \tau+A_{v}\end{array}$ \\
\hline
\end{tabular}

A class of $R_{3}$ or $U_{2}$ consists of all rotations by a given angle about any axis. The character of the $j$ th irreducible representation is $\left[\sin \left(j+\frac{1}{2}\right) \theta / \sin \theta / 2\right]$ where $j$

TABLE III. Polynomials $P$ for four- and five-dimensional representations of $I$ and $I_{h}$.

is integral for $R_{3}$ and integral or half-integral for $U_{2}$. The actual angle of rotation is $\theta$. The eigenvalues of any unimodular unitary irreducible representation matrix $D(j, \theta)$ in the class $\theta$ are $\exp (i j \theta), \exp [i(j-1) \theta]$, $\cdots, \exp (-i j \theta) .^{.}$The irreducible representation matrices for $U_{2} \times R_{3}$ are $D_{u}(S, \theta) \times D_{R}\left(l, \theta^{\prime}\right)$ and the eigenvalues of this are the products of the eigenvalues of $D_{u}$ and $D_{R}$ taken in all combinations. If we set $\xi=$ $\exp (i \theta)$ and $\eta=\exp \left(i \theta^{\prime}\right)$, these eigenvalues are $\xi^{k} \eta^{m}$ where $k=s, s-1, \cdots,-s$ and $m=l, l-1, \cdots,-l$. Hence the $R$ polynomial for this representation matrix is

$$
R(s, l)=\prod_{\boldsymbol{k}} \prod_{m}\left(\lambda \xi^{k} \eta^{m}+1\right) .
$$

It is convenient to change the notation to conform with that commonly used in the theory of atoms. Thus, $D_{u}(s, \theta) \times D_{R}\left(l, \theta^{\prime}\right)$ can be denoted ${ }^{2 o+1}(l)$, $l=S, P, D$, etc. in the standard fashion. Since among the one-electron spin orbitals $S=\frac{1}{2}$, we are actually interested only in the polynomial $R\left(\frac{1}{2}, l\right)$. It is ob-

${ }^{3}$ F. D. Murnaghan, The Theory of Group Representations (The Johns Hopkins Press, Baltimore, Md., 1938). 
SYM ETRY OF DETERMINANTAL WAVEFUNCTIONS

TABLE IV. Polynomials $R$ for two-dimensional representations of common point groups.

\begin{tabular}{|c|c|c|c|c|}
\hline Group & Species & $\left(\lambda^{4}+1\right)$ & $\left(\lambda^{3}+\lambda\right)$ & $\lambda^{2}$ \\
\hline$D_{3}, C_{8 v}$ & $E$ & ${ }^{1} A_{1}$ & ${ }^{2} E$ & ${ }^{8} A_{2}+{ }^{1} A_{1}+1 E$ \\
\hline$D_{4}, C_{4 v}$ & $E$ & ${ }^{1} A_{1}$ & ${ }^{2} E$ & ${ }^{8} A_{2}+{ }^{1} A_{3}+{ }^{1} B_{1}+{ }^{1} B_{2}$ \\
\hline \multirow[t]{2}{*}{$D_{6}, C_{5 v}$} & $E_{1}$ & ${ }^{1} A_{1}$ & ${ }^{2} E_{1}$ & ${ }^{8} A_{2}+{ }^{1} A_{1}+^{1} E_{2}$ \\
\hline & $E_{2}$ & ${ }^{1} A_{1}$ & ${ }^{\prime} E_{2}$ & ${ }^{8} A_{2}+{ }^{1} A_{1}+{ }^{1} E_{1}$ \\
\hline$D_{6}, C_{6 v}$ & $E_{i}$ & ${ }^{1} A_{1}$ & ${ }^{2} E_{i}$ & ${ }^{3} A_{2}+{ }^{1} A_{1},+^{1} E_{2}$ \\
\hline$D_{3 h}$ & $E^{(p)}$ & ${ }^{1} A_{1}{ }^{\prime}$ & ${ }^{2} E^{(p)}$ & ${ }^{3} A_{2}{ }^{\prime}+{ }^{1} A_{1}{ }^{\prime}+{ }^{1} E^{\prime}$ \\
\hline$D_{4 k}$ & $E_{o(u))}$ & ${ }^{1} A_{1 \sigma}$ & ${ }^{2} E_{g(u)}$ & ${ }^{3} A_{2 g}+{ }^{1} A_{1 \sigma}+{ }^{1} B_{1 \sigma}+{ }^{1} B_{2 g}$ \\
\hline \multirow[t]{2}{*}{$D_{5 h}$} & $E_{1}^{(p)}$ & ${ }^{3} A_{1}{ }^{\prime}$ & ${ }^{2} E_{1}(p)$ & ${ }^{3} A_{2}^{\prime}+{ }^{1} A_{1}^{\prime}+{ }^{1} E_{2}^{\prime}$ \\
\hline & $E_{2}(p)$ & ${ }^{1} A_{1}{ }^{\prime}$ & ${ }^{2} E_{2}(p)$ & ${ }^{3} A_{2}^{\prime}+{ }^{1} A_{1}^{\prime}+{ }^{1} E_{1}^{\prime}$ \\
\hline$D_{6 k}$ & $E_{i \sigma(u)}$ & ${ }^{1} A_{1 g}$ & ${ }^{2} E_{i \sigma(u)}$ & ${ }^{3} A_{2 v}+{ }^{1} A_{1 \sigma}+{ }^{1} E_{2 \sigma}$ \\
\hline$D_{2 d}$ & $E$ & ${ }^{1} A_{1}$ & ${ }^{2} E$ & ${ }^{8} A_{2}+{ }^{1} A_{1}+{ }^{1} B_{1}+{ }^{1} B_{2}$ \\
\hline$D_{3 d}$ & $E_{g(u)}$ & ${ }^{1} A_{10}$ & ${ }^{2} E_{g(u)}$ & ${ }^{3} A_{2 \sigma}+{ }^{1} A_{1 g}+{ }^{1} E_{\sigma}$ \\
\hline \multirow[t]{2}{*}{$D_{4 d}$} & $E_{i}(i=1,3)$ & ${ }^{1} A_{1}$ & ${ }^{2} E_{i}$ & ${ }^{3} A_{2}+{ }^{1} A_{1}+{ }^{2} E$ \\
\hline & $E_{2}$ & ${ }^{1} A_{1}$ & ${ }^{8} E_{2}$ & ${ }^{3} A_{2}+{ }^{1} A_{1}+{ }^{1} B_{1}+{ }^{1} B_{2}$ \\
\hline \multirow[t]{2}{*}{$D_{b d}$} & $E_{10(u)}$ & ${ }^{1} A_{1 o}$ & ${ }^{2} E_{1 g(u)}$ & ${ }^{3} A_{2 g}+{ }^{1} A_{1 \rho}+{ }^{1} E_{2 \sigma}$ \\
\hline & $E_{2 \rho(u)}$ & ${ }^{1} A_{1 \mathrm{~g}}$ & ${ }^{2} E_{2 \rho(u)}$ & ${ }^{8} A_{2 \sigma}+{ }^{1} A_{1 \sigma}+{ }^{1} E_{1 \sigma}$ \\
\hline \multirow[t]{3}{*}{$D_{b d}$} & $E_{i}(i=1,5)$ & ${ }^{1} A_{1}$ & ${ }^{2} E_{i}$ & ${ }^{3} A_{2}+{ }^{1} A_{1}+{ }^{1} E_{2}$ \\
\hline & $E_{i}(i=2,4)$ & ${ }^{1} A_{1}$ & ${ }^{2} E_{i}$ & ${ }^{8} A_{2}+{ }^{1} A_{1}+{ }^{1} E_{4}$ \\
\hline & $E_{s}$ & ${ }^{1} A_{1}$ & ${ }^{2} E_{8}$ & ${ }^{\mathrm{s}} A_{2}+{ }^{1} A_{1}+{ }^{1} B_{1}+{ }^{1} B_{2}$ \\
\hline$T_{d}, O$ & $E$ & ${ }^{1} A_{1}$ & ${ }^{2} E$ & ${ }^{3} A_{2}+{ }^{1} A_{1}+{ }^{1} E$ \\
\hline$O_{h}$ & $E_{q(u)}$ & ${ }^{1} A_{1} \rho$ & ${ }^{2} E_{g(u)}$ & ${ }^{3}{ }_{2 q}+{ }^{1} A_{1 q}+{ }^{1} E_{q}$ \\
\hline$C_{\infty v}$ & $\Lambda$ & ${ }^{1} \Sigma^{+}$ & ${ }^{2} \Lambda$ & ${ }^{3} \Sigma^{-}+{ }^{1} \Sigma^{+}+{ }^{1}(2 \Lambda)$ \\
\hline$D_{\text {onh }}$ & $\Lambda_{g(u)}$ & ${ }^{1} \Sigma_{g}+$ & ${ }^{2} \Lambda_{\theta(u)}$ & ${ }^{3} \Sigma_{\sigma}-{ }^{-1} \Sigma_{g}++^{1}(2 \Lambda)_{\sigma}$ \\
\hline
\end{tabular}

TABLE V. Polynomials $R$ for the three-dimensional representations of $T, T_{d}, T_{h}, O, O_{h}, I$, and $I_{h}$.

\begin{tabular}{|c|c|c|c|c|c|}
\hline Group & Species & $\left(\lambda^{6}+1\right)$ & $\left(\lambda^{5}+\lambda\right)$ & $\left(\lambda^{4}+\lambda^{2}\right)$ & $\lambda^{3}$ \\
\hline$T$ & $F$ & ${ }^{1} A$ & ${ }^{2} F$ & ${ }^{8} P+2^{\prime} F$ & $4 A+2^{2} F+{ }^{2} E$ \\
\hline$T_{h}$ & $F_{\sigma(u)}$ & ${ }^{1} A_{\theta}$ & ${ }^{2} F_{g(u)}$ & ${ }^{3} F_{q}+2^{1} F_{\theta}$ & $4 A_{\theta(u)}+2^{2} F_{o(u)}+{ }^{2} E_{g(u)}$ \\
\hline$T_{d}, O$ & $F_{i}$ & ${ }^{1} A_{1}$ & ${ }^{2} F_{i}$ & ${ }^{8} F_{1}+{ }^{1} A_{1}+{ }^{1} E+{ }^{\prime} F_{2}$ & ${ }^{4} A_{i}+{ }^{2} E+{ }^{2} F_{1}+{ }^{2} F_{2}$ \\
\hline$o_{h}$ & $F_{i g(u)}$ & ${ }^{1} A_{10}$ & ${ }^{2} F_{i g(u)}$ & ${ }^{8} F_{i q}+{ }^{1} A_{1 q}+{ }^{1} E_{q}+{ }^{1} F_{2 g}$ & ${ }^{4} A_{i g(u)}+{ }^{2} E_{g(u)}+{ }^{2} F_{i g(u)}+{ }^{2} F_{2 g(u)}$ \\
\hline$I$ & $F_{i}$ & ${ }^{1} A$ & ${ }^{2} F_{i}$ & ${ }^{8} F_{i}+{ }^{1} A+{ }^{1} H$ & ${ }^{4} A+{ }^{2} F_{i}+{ }^{2} H$ \\
\hline$I_{\boldsymbol{k}}$ & $F_{i o(u)}$ & ${ }^{1} A_{v}$ & ${ }^{2} F_{i g(u)}$ & ${ }^{3} F_{i q}+{ }^{3} A_{q}+{ }^{1} H_{q}$ & ${ }^{4} A_{\theta(u)}+{ }^{2} F_{i g(u)}+{ }^{2} H_{\theta(\psi)}$ \\
\hline
\end{tabular}


TABLE VI. Polynomials $R$ for four- and five-dimensional representations of $I$ and $I_{\hbar}$.

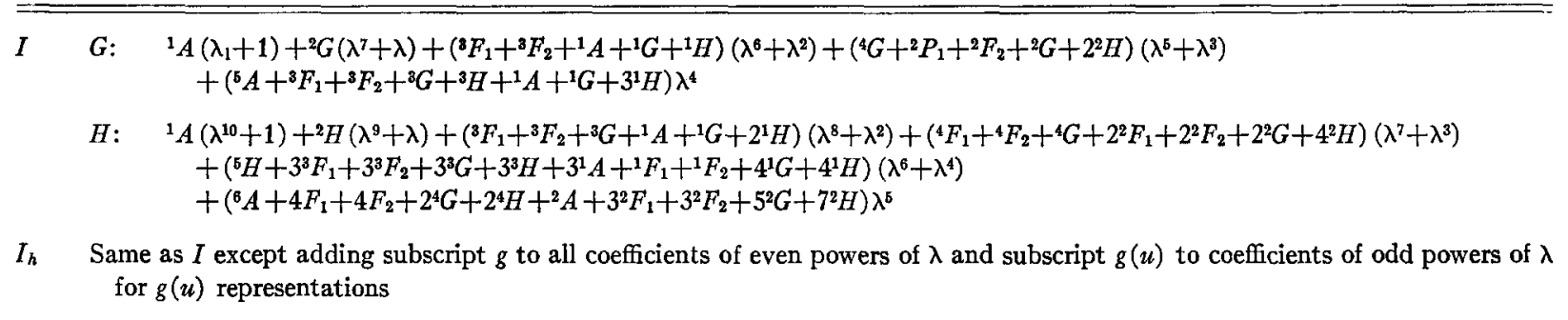

served that

$$
\begin{aligned}
& {\left[R\left(\frac{1}{2}, l+1\right) / R\left(\frac{1}{2}, l\right)\right]} \\
& =G_{l+1}(\lambda) \\
& =\left(\lambda^{4}+1\right)+\left(\xi^{3}+\xi^{-\frac{1}{2}}\right)\left(\eta^{l+1}+\eta^{-l-1}\right)\left(\lambda^{3}+\lambda\right) \\
& \quad+\left[\left(\xi+1+\xi^{-1}\right)+1+\eta^{2 l+2}+\eta^{-2 l-2}\right] \lambda^{2} .
\end{aligned}
$$

It is easily seen that $G_{0}(\lambda)=\left(\lambda^{2}+1\right)+\left(\xi^{\frac{1}{2}}+\xi^{\frac{1}{2}}\right) \lambda$; hence,

TABLE VII. The polynomials $G_{l}(\lambda)$ for atomic states.

$$
\begin{aligned}
& G_{0}^{e}={ }^{1} S^{e}\left(\lambda^{2}+1\right)+{ }^{2} S^{e} \lambda \\
& G_{1}{ }^{(0, e)}={ }^{1} S^{e}\left(\lambda^{4}+1\right)+\left({ }^{2} D^{(0, e)}-{ }^{2} S^{(0, e)}\right)\left(\lambda^{3}+\lambda\right) \\
& +\left({ }^{3} S^{e}+{ }^{1} S^{e}+{ }^{1} D^{e}-1 P^{e}\right) \lambda^{2} \\
& G_{2}{ }^{(0, e)}={ }^{1} S^{e}\left(\lambda^{4}+1\right)+\left({ }^{2} D^{(0, e)}-{ }^{2} P^{(0, e)}\right)\left(\lambda^{3}+\lambda\right) \\
& +\left({ }^{3} S^{e}+{ }^{1} S^{e}+{ }^{1} G^{e}-{ }^{1} F^{e}\right) \lambda^{2} \\
& G_{3}(0, e)={ }^{1} S^{e}\left(\lambda^{4}+1\right)+\left({ }^{2} F^{(0, e)}-{ }^{2} D^{(0, e)}\right)\left(\lambda^{8}+\lambda\right) \\
& +\left({ }^{3} S^{e}+{ }^{1} S^{e}+{ }^{1} K^{e}-1 H^{e}\right) \lambda^{2}
\end{aligned}
$$

we may write

$$
R\left(\frac{1}{2}, l\right)=\prod_{k=0}^{l} G_{k}(\lambda)
$$

It is observed that $G_{l}$ may also be written as

$$
G_{l}={ }^{1} S\left(\lambda^{4}+1\right)+\left[{ }^{2}(l)-{ }^{2}(l-1)\right]\left(\lambda^{3}+\lambda\right)
$$

$$
+\left[{ }^{3} S+{ }^{1} S+{ }^{1}(2 l)-{ }^{1}(2 l-1)\right] \lambda^{2} \text {. }
$$

If we had used the three-dimensional real orthogonal group $\mathrm{O}_{3}$ instead of $R_{3}$, the irreducible representations can be classified as odd or even, and since the space part of the individual spin orbitals is odd or even with $l$, this may be included in the calculation easily. Table VII gives the first few $G$ polynomials in terms of Russell-Saunders term symbols. It may be pointed out that a simple necessary condition for the correctness of the results is the disappearance of all minus signs in $R$ in spite of their presence in the $G_{l}$. 